\title{
Validated or not validated? Is that the question?
}

This BJD issue contains a thought-provoking systematic review article.(1) In it, the authors reviewed the patient-reported outcome measures (PROMs) available for use in facial skin cancer, and then conducted quality assessments of them. The assessments included using two systems: the Consensus-based Standards for the Selection of Health Status Measurement Instruments (COSMIN),(2) and those proposed by Terwee and colleagues.(3) The first of these informs the choice of analyses to most appropriately study different psychometric properties, and the second also specifies what constitutes desirable results. The psychometric performance of the PROM under investigation can be compared to these targets. Other similar frameworks exist, though the choice is probably of less interest than considering the implications of the analyses and results presented by Dobbs and colleagues.

The review is well-designed and conducted, and several points of discussion follow from reading it. Firstly, psychometric properties cannot reasonably be reduced to something present or absent in a binary sense, as is suggested by statements like "a validated PROM was used...". Indeed, validity itself is an umbrella term encompassing a range of elements, before even introducing other psychometric properties like reliability and responsiveness. For a particular PROM deployed in a specific clinical situation, all, some, or none, of the desirable psychometric properties may have been studied and demonstrated. As was largely found in the review, it is common for only some psychometric properties to have been studied. Deciding how to proceed in such a scenario can be challenging. When designing a trial for example, should such a PROM be used? Or, if appraising published research using the PROM, was it fit for purpose?

These issues might be resolved in different ways. Here, the authors use the OMERACT filter to determine a "bottom line".(4) This is reasonable in a review article, but might oversimplify the matter in some real-world applications. Alternatively, prioritising different psychometric properties could support situation-specific solutions. For example, it may be that having item content with high face validity is the priority if choosing a PROM to support clinical consultations. In contrast, having interval-level measurement via item response theory-based structural validity may be more important in quantitative research.

Furthermore, psychometric evidence gaps might be resolved by extrapolating evidence from other clinical areas. In this review, the authors structured their search to specifically identify facial skin cancer studies. A broader approach may have yielded more evidence of validity for the PROMs examined. 
Whether it is appropriate to extrapolate psychometric property evidence from one clinical context to another may depend on the underlying construct being measured. In this review, that could be "skin cancer morbidity", or "facial appearance and function", or "health-related quality of life", among others. If the construct of interest were skin cancer morbidity per se, then studies validating a PROM in skin cancer elsewhere on the body may still provide useful evidence.

Many of these issues are subjective and may be context-specific. Ultimately, this systematic review not only summarises the current state of PROMs for facial skin cancer work, but also reminds each of us to consider the robustness and suitability of outcome measures in all areas of clinical practice, research and critical appraisal.

Conflicts of interest:

The author currently collaborates with the primary author of the systematic review referenced in this commentary on other projects but has no current or past affiliations or other involvement in any organisation or entity with an interest in this commentary. The author is funded by the NIHR. The views expressed are the author's own, and are not necessarily those of the NIHR, NHS or the Department of Health and Social Care.

Author:

Jeremy Rodrigues

NIHR Postdoctoral Fellow

Nuffield Department of Orthopaedics, Rheumatology and Musculoskeletal Sciences (NDORMS), University of Oxford, Oxford, UK orcid.org/0000-0002-9347-5026

Email: Jeremy.rodrigues@ndorms.ox.ac.uk

\section{References}

1. Dobbs TD, Samarendra H, Hughes S, Hutchings HA, Whitaker I. Patient reported outcome measures for facial skin cancer: A systematic review and evaluation of the quality of their measurement properties. British Journal of Dermatology. 2019; In press.

2. Mokkink LB, Terwee CB, Knol DL, Stratford PW, Alonso J, Patrick DL, et al. The COSMIN checklist for evaluating the methodological quality of studies on measurement properties: a clarification of its content. BMC Med Res Methodol. 2010;10:22. 
3. Terwee CB, Bot SDM, de Boer MR, van der Windt DAWM, Knol DL, Dekker J, et al. Quality criteria were proposed for measurement properties of health status questionnaires. J Clin Epidemiol. 2007;60:34-42.

4. Boers M, Brooks P, Strand CV, Tugwell P. The OMERACT filter for Outcome Measures in Rheumatology. J Rheumatol. 1998;25(2):198-9. 